\title{
Velocity Profile inside Piezoacoustic Inkjet Droplets in Flight: Comparison between Experiment and Numerical Simulation
}

\author{
Arjan van der Bos, ${ }^{1}$ Mark-Jan van der Meulen, ${ }^{2}$ Theo Driessen, ${ }^{2}$ Marc van den Berg, ${ }^{1}$ Hans Reinten, ${ }^{1}$ \\ Herman Wijshoff, ${ }^{1}$ Michel Versluis, ${ }^{2}$ and Detlef Lohse ${ }^{2}$ \\ ${ }^{1}$ Océ Technologies, P.O. Box 101, 5900 MA Venlo, The Netherlands \\ ${ }^{2}$ Physics of Fluids group and MESA ${ }^{+}$Institute for Nanotechnology, Faculty of Science and Technology, \\ University of Twente, P.O. Box 217, 7500 AE Enschede, The Netherlands
}

(Received 12 December 2013; published 27 February 2014)

\begin{abstract}
Inkjet printing deposits droplets with a well-controlled narrow size distribution. This paper aims at improving experimental and numerical methods for the optimization of drop formation. We introduce a method to extract the one-dimensional velocity profile inside a single droplet during drop formation. We use a novel experimental approach to capture two detailed images of the very same droplet with a small time delay. The one-dimensional velocity within the droplet is resolved by accurately determining the volume distribution of the droplet. We compare the obtained velocity profiles to a numerical simulation based on the slender jet approximation of the Navier-Stokes equation and we find very good agreement.
\end{abstract}

DOI: 10.1103/PhysRevApplied.1.014004

\section{INTRODUCTION}

Inkjet printing is well known for its impressive reproducibility of the drop formation [1-3]. This has made inkjet printing technology a reliable technique for drop deposition of liquids for a broad range of applications [4,5], owing to the increase of the deposition accuracy and further reduction of the droplet sizes $[6,7]$.

Numerical models for inkjet printing, e.g., finite element, finite difference, and boundary integral methods, are used to develop printheads for these applications. One very successful model is the slender jet approximation of the Navier-Stokes equation, often also denoted as lubrication approximation. It has been shown to be fast and reliable for modeling of drops [8,9] and sprays [10] and incorporates breakup dynamics and coalescence of the drops (Driessen et al. [11]). The low-CPU time requirements of such a model, which are of the order of minutes on a personal computer, allows for rapid exploration of a large parameter space in a short time.

As a result of high droplet velocities and decreasing droplet volumes, the experimental validation of the numerical models becomes increasingly challenging $[1,3,12]$. Visualizing fast inkjet droplets requires ultra-high-speed cameras imaging at frame rates exceeding one million frames per second [10,13-15], or stroboscopic techniques with very short illumination times $(<20 \mathrm{~ns})$ to freeze the motion in the droplets $[1,2,16]$. Even then the results are mostly limited to global estimates such as the overall drop velocity, drop volume, and number of satellites. In contrast, numerical models reveal detailed local information on tail formation, velocity development, and fluid dynamics throughout the entire drop formation.
In this paper we present an experimental method that reaches beyond current ones; we will extract the local and global droplet dynamics during the entire formation of a single picoliter sized droplet. We introduce an advanced imaging technique that provides two snapshot images of the very same droplet with extremely high temporal and spatial resolution. The image pair is analyzed by extracting the contour of the droplet, and subsequently by calculating the volume distribution over the droplet. The one-dimensional velocity inside the droplet is given by the small displacements in the volume distribution over the time between the two recordings.

Our method allows for a fully quantitative comparison of the measured drop dynamics with the numerical models. Here we compare the experimental data with the results obtained from the lubrication approximation. The volume and velocity distribution obtained from experiment are used as initial condition for the numerical simulation. The time evolution of the droplet can then be used to validate both the experimental and numerical approach.

\section{EXPERIMENTAL SETUP}

Figure 1 shows a schematic overview of the experimental setup, which consists of essentially four components: printhead, light source, imaging system, and timing control hardware.

The inkjet printhead is developed by Océ Technologies [17-20]. The piezoacoustic inkjet printhead contains 256 similar ink channels with an inverted trumpet shape nozzle with an opening radius of $15 \mu \mathrm{m}$. The printhead is distinguished by a very reproducible drop formation $[3,17]$ and negligible angular distortion with respect to 


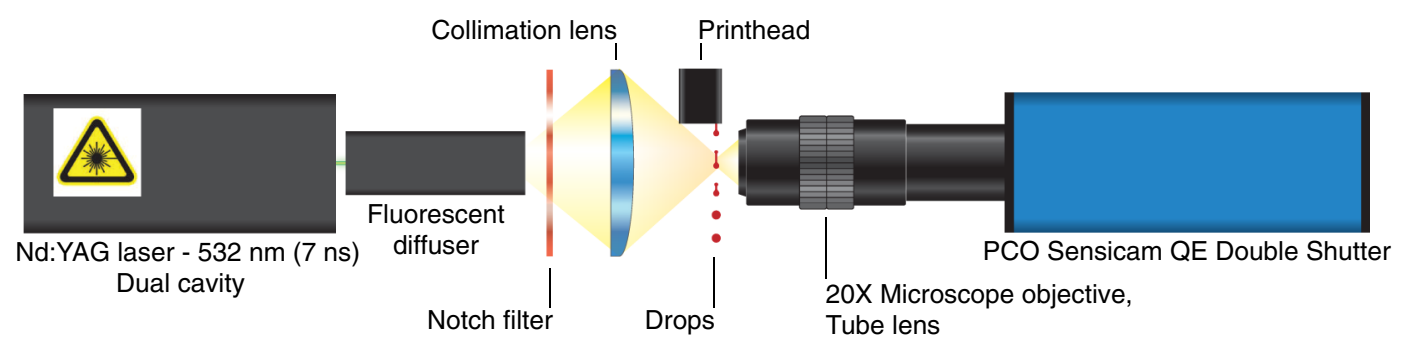

FIG. 1 (color online). In the experimental setup, the laser pulse from the Nd:YAG laser illuminates the diffusor, which emits a fluorescent light pulse of $8 \mathrm{~ns}$ at high intensity. The fluorescence is focused by an aspherical condenser lens onto the droplets. A notch filter, placed between the condenser lens and the droplets, prevents any remaining laser light from reaching the CCD camera. The images are recorded using an inline assembly microscope with a dual frame camera.

jetting direction. Applying an actuation pulse of $30 \mathrm{~V}$ to the piezoelectric actuator results in $11 \mathrm{pl}$ droplets with a final velocity of $4 \mathrm{~m} / \mathrm{s}$. Much higher velocities, up to $15 \mathrm{~m} / \mathrm{s}$, occur during the formation of the droplet at the meniscus and in the tail of the droplet.

The experiments were conducted with silicone oil, which has several advantages. First, the temperature dependence of the viscosity is less than $1 \% / \mathrm{K}$ and silicone oil therefore acts as a Newtonian liquid. Second, the surface tension of silicone oil is not easily affected by contamination, hence the surface tension can be assumed to be constant during the drop formation. The silicone oil that was used is AK10 of Wacker-Chemie $\mathrm{GmbH}$, which has a viscosity of $9.3 \mathrm{mPas}$, a surface tension of $20.2 \mathrm{mN} / \mathrm{m}$, and a density of $930 \mathrm{~kg} / \mathrm{m}^{3}$.

To illuminate the droplets, we use a dual-cavity Nd:YAG laser $(\lambda=532 \mathrm{~nm}$, Litron Nano-S). The laser creates a $7 \mathrm{~ns}$ laser pulse with high intensity (65 $\mathrm{mJ}$ per pulse), which is coupled into a fluorescent diffuser. The fluorescent light generated in the diffuser remains short (approximately $8 \mathrm{~ns}$ ) and intense, while both the temporal and spatial coherence have been lost [21-23]. This makes it highly suitable for imaging purposes without speckle and interference fringes.
The residual laser light, which could distort the image or even damage the CCD sensor, is removed with a notch filter. To ensure optimal coupling into the imaging system, the fluorescent light is collimated with an aspherical lens such that the numerical aperture closely matches that of the microscope $(\mathrm{NA}=0.4)$ [24].

To image the picoliter sized droplets a microscope is connected to a dual-frame PIV camera (PCO Sensicam QE Double Shutter), capable of capturing two consecutive images with a delay as low as $500 \mathrm{~ns}$ in between. The microscope is an inline assembly microscope (Infini Tube ${ }^{\mathrm{TM}}$ ) equipped with a $20 \times$ objective with a NA of 0.42 (Edmund Optics, M-Plan-APO). This results in an imaging resolution of approximately $365 \mathrm{~nm} /$ pixel. Because of the imperfection of the optics the resolution is not exactly constant over the whole field of view. A correction for this "pincushion" has been applied to the images. This correction is determined using a highly accurate calibration grid. Figure 2 shows a time series of drop formation, illustrating the typical high-quality images obtained with our system.

The timing is controlled by a high-precision delay generator (BNC 575, Berkeley Nucleonics) which triggers

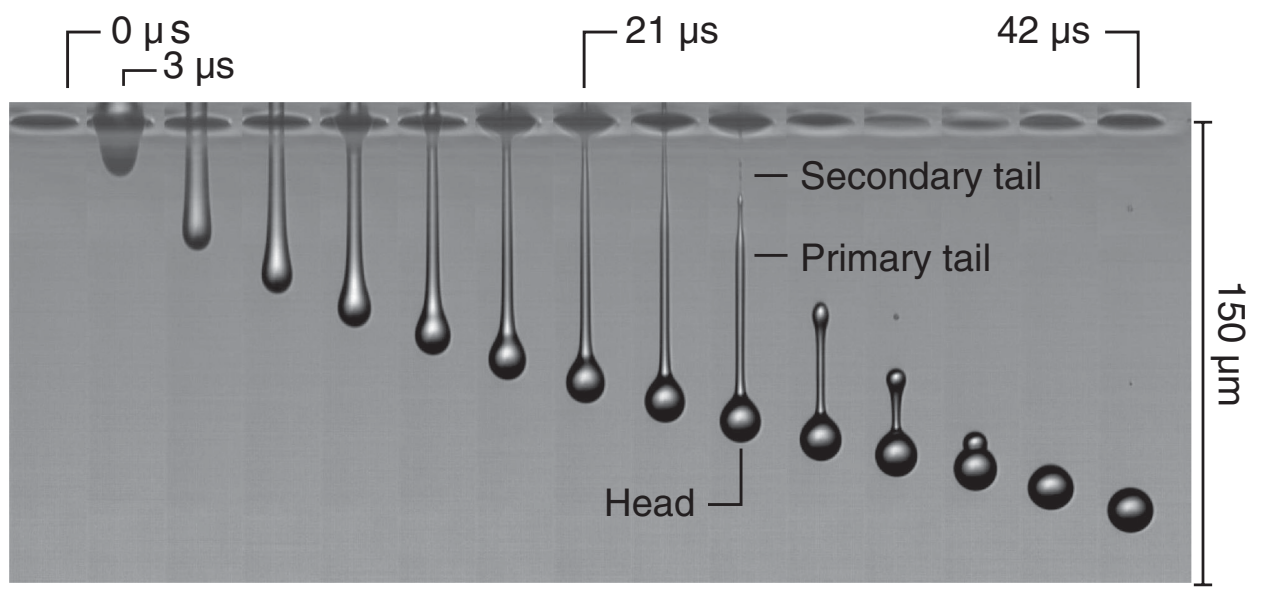

FIG. 2. Time series of droplets recorded with single-flash photography. From left to right, multiple images of single droplets with a delay of $3 \mu$ s between these individual droplets. The width of the droplet is $23 \mu \mathrm{m}$, the tail is about $4 \mu \mathrm{m}$, and the secondary tail has a width below $1 \mu \mathrm{m}$. The figure illustrates the imaging quality of the setup, and the absence of motion blur due to the use of the $8 \mathrm{~ns}$ iLIF. 
the camera, laser, and printhead. The illumination time and the delay between two flashes is verified using a high-speed photodetector (Thorlabs DET 210) which has a typical rise and fall time of $1.2 \mathrm{~ns}$.

\section{EXPERIMENTAL METHODS}

\section{A. High-resolution imaging of drop formation}

Imaging drop formation in inkjet printing with high detail is very challenging, as the micron-sized droplets move with a velocity of several meters per second. It requires a high degree of spatial and temporal resolution, which is offered by a combination of large high optical quality imaging systems with sensitive high-resolution cameras. It is convenient to use a camera with a small pixel size to keep the magnification to a minimum (as it may severely distort the image quality).

Equally important is the time duration that the CCD sensor is exposed. This time duration can be the exposure time of the camera or, in case of a short flash, the duration of the flash. The exposure needs to be optimized such that sufficient contrast is obtained while minimizing the motion blur. The following criterion (Ref. [23]) is used for the maximum allowable exposure time $(\tau)$ :

$$
\tau \leq \frac{\text { pixel size }}{u \cdot M_{\text {eff }}},
$$

where $u$ is the velocity of the droplet. The spatial resolution of the images is given by the magnification of the optics $\left(M_{\text {eff }}\right)$ and the pixel size of the CCD sensor. In our experiment, the fastest fluid element is displaced with a velocity of $15 \mathrm{~m} / \mathrm{s}$. Following Eq. (1) the maximal allowable illumination time is $18 \mathrm{~ns}$. Here we use a flash of approximately $8 \mathrm{~ns}$, thus for the present setup the motion blur is reduced to less than half the size of a pixel.

\section{B. Dual imaging of drop formation in flight}

As inkjet printing is very reproducible, stroboscopic imaging or single flash imaging would suffice for a statistical analysis. Here the aim is to visualize the dynamics during drop formation, for which the reproducibility is not sufficient; this requires at least two images of the very same droplet. Although it may seem straightforward to use time-resolved high-speed imaging techniques, the time and length scales in this problem would require ultra-high-speed imaging systems running at several million frames per second, e.g., the Brandaris 128 camera $[15,25]$, with the added complexity of optical configuration, triggering, and illumination.

Our approach creates a time series of the entire drop formation by shifting the delay of the flash with respect to the start of drop formation. As a result of the high degree of reproducibility of the inkjet system, the result of such a stroboscopic technique is very similar to a time series obtained in time-resolved high-speed imaging [14,15]. Here we add functionality by recording two consecutive images of one single droplet at each time step with the double shutter camera and the dual-cavity laser. It is key to have the time delay $\left(\tau_{f}\right)$ between the two consecutive images, denoted as frame A and frame B, such that the lowest possible detectable displacement is obtained. The delay is limited in two ways. Its lower limit is given by hardware limitations of the camera, here $500 \mathrm{~ns}$. The upper limit comes from a fluid dynamical point of view. For the determination of the velocity along the axis of symmetry of the droplet, we assume that the motion is dominated by the inertia of the fluid. To meet this assumption, $\tau_{f}$ must be smaller than the viscocapillary time scale $\left(\tau_{\mathrm{vc}}\right)$, given by

$$
\tau_{\mathrm{vc}}=\frac{\mu R}{\sigma} .
$$

In the experiments we use silicone oil with a viscosity of $9.3 \mathrm{mPas}$, and a surface tension of $20.2 \mathrm{mN} / \mathrm{m}$. As the tail behind the head of the droplet has a width of approximately $10 \mu \mathrm{m}$, this gives an upper limit of $\tau_{\mathrm{vc}}$ of approximately $2 \mu \mathrm{s}$.

\section{IMAGE PROCESSING}

The experimental setup delivers detailed high-resolution images, from which the volume distribution of the droplet is determined. It requires accurate subpixel edge detection, independent of the contrast of the image. Part of the printhead blocks the illumination, causing an uneven intensity distribution in the images. In addition, the intensity of the light source fluctuates approximately 4\%. With standard (fast) edge detection techniques the precise location of the edge is very sensitive to these fluctuations. This problem is solved by using a two-pass edge detection method, illustrated in Fig. 3. The first pass consists of a conventional contour tracing routine (Mathworks Matlab). The second pass determines the intensity curve along the normal of the contour. By calculating the inflection point [26] along these normal lines the intensity independent contour is found.

To verify the validity of this method, a spherical droplet is imaged at a constant delay $\left(t_{d}\right)$ while gradually increasing the background intensity of the image within the dynamic range of the camera. From each of the images the radius of the droplet is calculated using conventional contour tracing and using the proposed method. Figure 4 shows the drop radius for increasing background intensity. This shows that, while the conventional method proves unreliable, our proposed method performs very well over the entire dynamic range of the camera. Only when the background is overexposed the method fails. This method allows for an accurate intensity independent contour determination. 

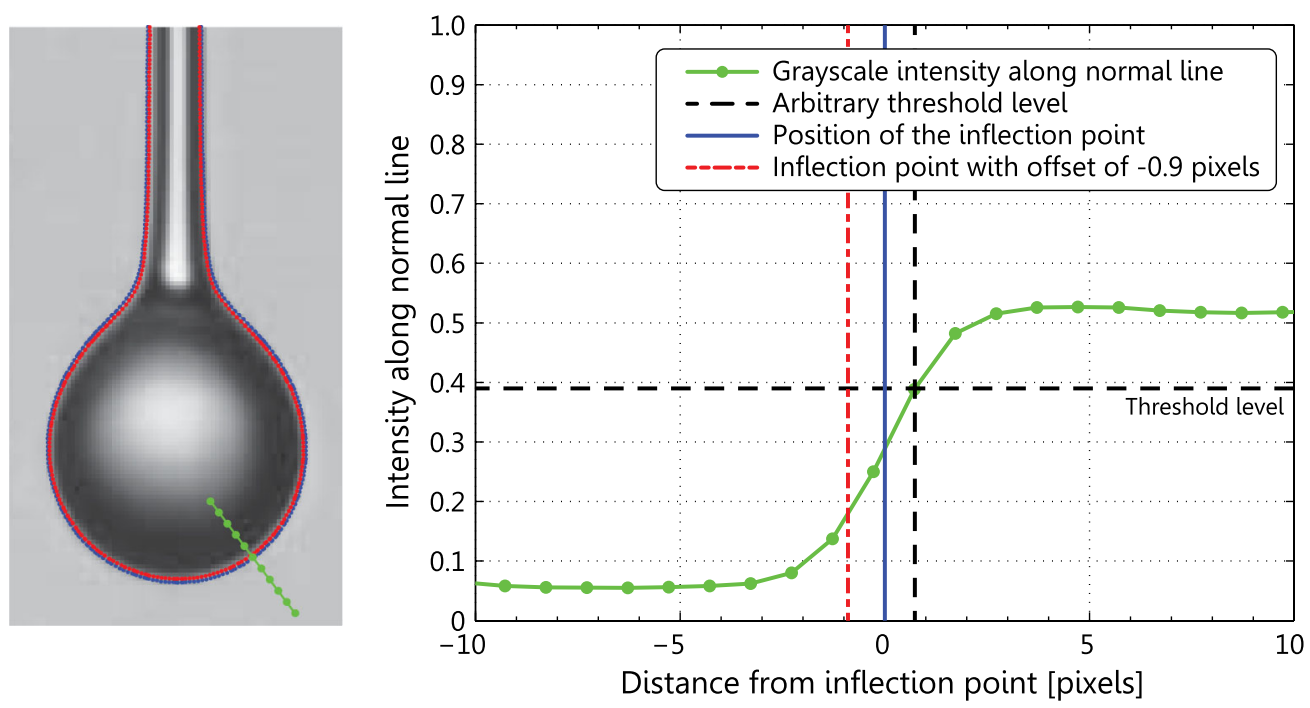

FIG. 3 (color online). Accurate subpixel edge detection, independent of the contrast in the image, is required. On the left a region of interest of the drop image is shown. First, the edge is determined using a standard threshold method (data not shown). From this trace the normal lines along the boundary of the drop are calculated (dotted green line). By calculating the inflection point along the normal lines the intensity independent contour is determined (blue). The red line shows the corrected position of the surface, which is determined by volume conservation arguments, see Sec. V. The right part shows the intensity curve along the normal line (green dotted line).

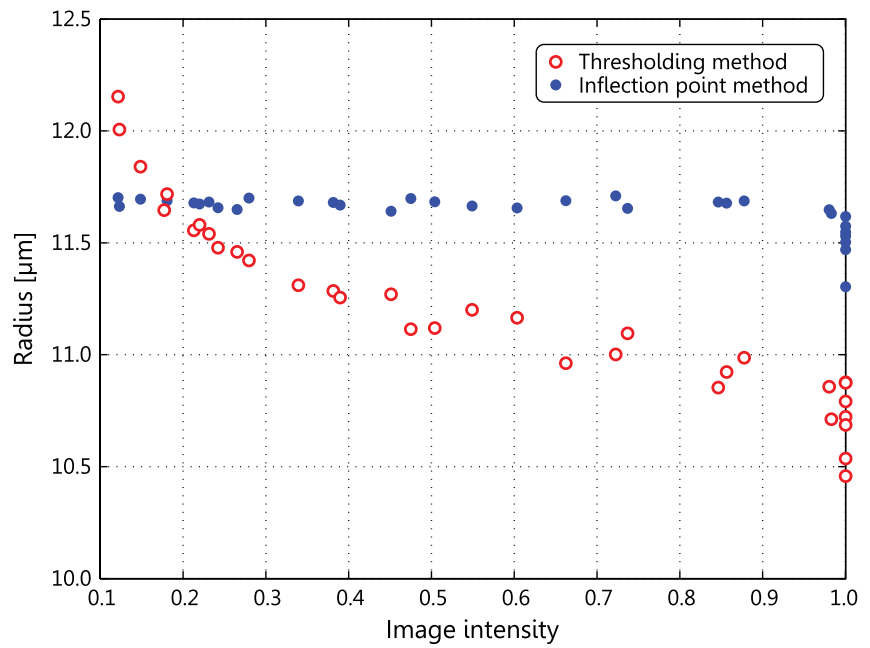

FIG. 4 (color online). A spherical droplet is imaged while gradually increasing the intensity of the illumination over the dynamic range of the camera. The radius is determined using a threshold method (red open dots) and by calculating the inflection point along the normal lines (blue filled dots). The radii from the threshold images change by $20 \%$ for increasing intensity, while the proposed method shows less than $1 \%$ variation. The remaining variation is primarily caused by the residual variation in the reproducibility of the drop formation (as shown in the inset of Fig. 6)

\section{DROPLET VOLUME}

From the contours of the droplet the volume of this droplet can be determined, assuming axial symmetry around the central axis of the droplet. Here we define the radius as the perpendicular distance between the central axis and the edge of the droplet, and is given by $R\left(x, t_{d}\right)$, where $x$ is the axis of symmetry, and $t_{d}$ is the time with respect to the start of the drop formation (Fig. 5). The volume of a droplet is calculated by integration over $x$,

$$
V_{\mathrm{drop}}\left(t_{d}\right)=\pi \int_{x_{0}}^{x_{\text {tip }}} R\left(x, t_{d}\right)^{2} d x
$$

Here $x_{0}$ is the nozzle position and $x_{\text {tip }}$ the tip of the drop. Because of the subpixel edge detection, the discrete $x$ values were resampled into nonequidistant values. The sampling resolution $d x$ has become so small that we can safely assume that the radius varies linearly over $d x$. Hence, the volume of each element becomes a truncated cone with

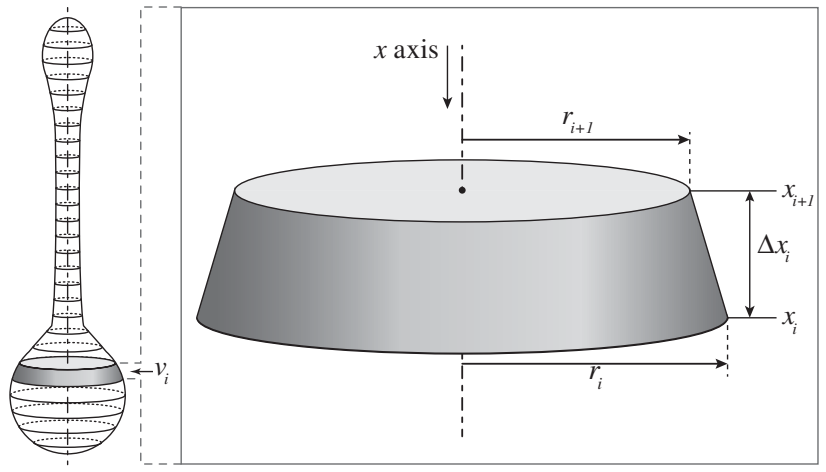

FIG. 5. For each droplet the contour was determined with subpixel accuracy. To calculate the volume distribution the drop is assumed to be axisymmetric. The volume $\left(v_{i}\right)$ of element $i$ of the drop is estimated by truncated cones with radii $r_{i}$ and $r_{i+1}$ and a height $\Delta x_{i}$ 
a finite width of $\Delta x_{i}$ (illustrated in Fig. 5), where each cone has a volume

$$
v_{i}=\pi \frac{\left(r_{i}^{2}+r_{i+1}^{2}+r_{i} r_{i+1}\right)}{3} \Delta x_{i}
$$

This gives the total drop volume at time $t_{d}$,

$$
V_{\mathrm{drop}}\left(t_{d}\right)=\pi \sum_{i=0}^{i=N} \frac{\left(r_{i}^{2}+r_{i+1}^{2}+r_{i} r_{i+1}\right)}{3} \Delta x_{i} .
$$

This method is used to determine the volumes of all droplets in a time series, as shown in Fig. 6. The figure shows that, as the droplet emerges at $16 \mu$ s from the nozzle, the volume starts to increase. At $60 \mu \mathrm{s}$ the droplet detaches from the meniscus, after which, for increasing delay, the shape of the detached droplet changes from elongated to spherical. The blue data show the volume, assuming the physical edge at the inflection point, as described in the previous section. The figure shows that for this data the volume increases over time. As evaporation is negligible and the drop formation is extremely reproducible, the actual volume of the detached droplet must remain constant. The origin of this overestimation lies in the physical surface not being at the inflection point. To compensate for

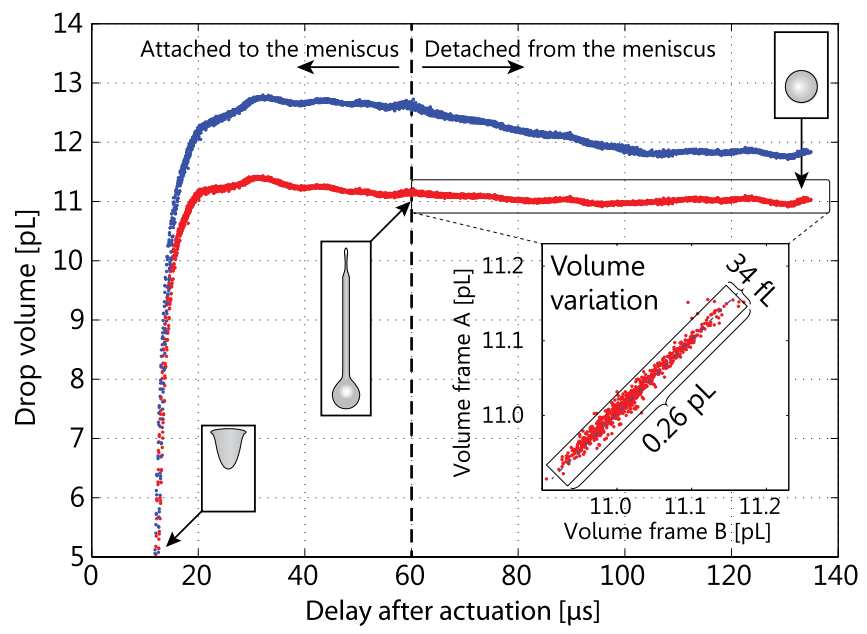

FIG. 6 (color online). The total volume $V_{\text {drop }}$ of each ejected droplet, as extracted from the recordings, given as a function of the delay time. For each delay the information of two image pairs, i.e., four images, are used. The second pair is recorded one full period after the first to confirm stability at longer time scale. After a delay of $10 \mu$ s the meniscus starts moving outward, and at approximately $60 \mu$ s the droplets are detached from the meniscus. The blue data give the uncorrected volume following the inflection point method, while the red data give volume after correcting using volume conservation. The inset shows the calculated corrected volume in frame $\mathrm{A}$ against the corrected volume in frame $\mathrm{B}$, which illustrates the volume reproducibility $\left(V_{\text {drop }}=11.02 \pm 0.13 \mathrm{pl}\right)$ and the accuracy of the method $(3 \sigma=17 \mathrm{fl})$. this inaccuracy, an inward shift of $330 \mathrm{~nm}$, with respect to the inflection point along the normal, is introduced to the edge detection method. Figure 6 (red data) confirms the accomplishment of the correction, as the volume remains constant over time. To confirm that the stability is time independent, the figure displays two full drop evolutions on top of each other, recorded at different times.

By displaying the volume in frame A against the volume in frame B over a time series (inset in Fig. 6), it is possible to estimate both the accuracy of the method and the reproducibility of drop formation. Here we find a droplet reproducibility of $V_{0}=11.02 \pm 0.13 \mathrm{pl}$. For a single drop the perpendicular distance from the linear regression line is

$$
\Delta V_{\mathrm{drop}}\left(t_{d}\right)=\frac{V_{\mathrm{drop}}\left(t_{d}\right)_{B}-V_{\mathrm{drop}}\left(t_{d}\right)_{A}}{\sqrt{2}} .
$$

Here $V_{\mathrm{drop}}\left(t_{d}\right)_{A}$ is the drop volume for frame $\mathrm{A}$, and $V_{\text {drop }}\left(t_{d}\right)_{B}$ is the drop volume of the same droplet in frame B. By calculating three times the standard deviation $(3 \sigma)$ over all volumes we find the accuracy of the method, here $3 \sigma=17 \mathrm{fl}$.

\section{DROPLET VELOCITY}

To determine the one-dimensional velocity profile inside the droplet at time $t_{d}$, the displacement of the volume elements $\left(v_{i}\right)$ is determined. Two images are captured for every single droplet; frame $\mathrm{A}$ at a drop delay $t_{d}$ and frame $\mathrm{B}$ at $t_{d}+\tau_{f}$. Here the time difference between the two frames $\left(\tau_{f}\right)$ was set to $600 \mathrm{~ns}$. First, the volume distribution is determined for both frames individually. Then, starting at the tip of the drop, the volume of each element in frame A is mapped to the volume elements in frame B (illustrated in Fig. 7). Here we define $\bar{x}_{A}(i)$ as the center of mass of the volume element in frame $\mathrm{A}$, and $\bar{x}_{B}(i)$ as its mapped

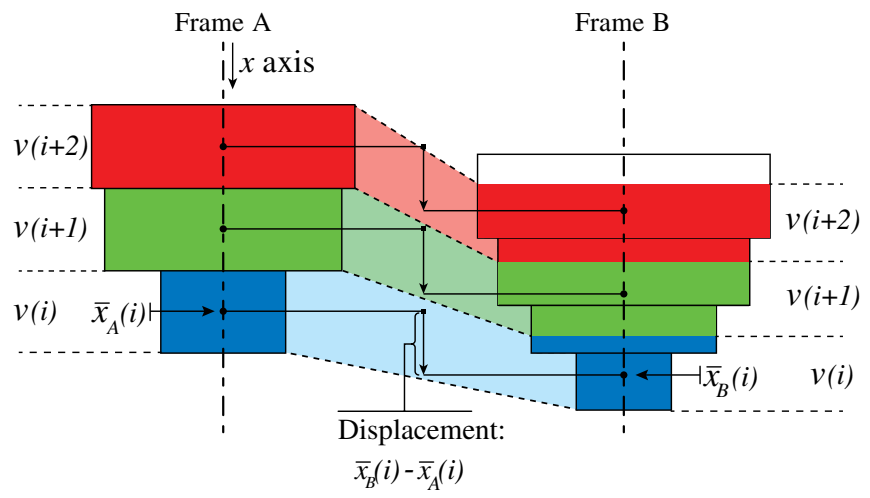

FIG. 7 (color online). The volume distribution is calculated for frames A and B. The volume per element of the first time step is mapped to the volume elements in the second time step. The displacement of the corresponding center of masses divided by the time interval between the recordings gives the mean velocity of the volume elements. 


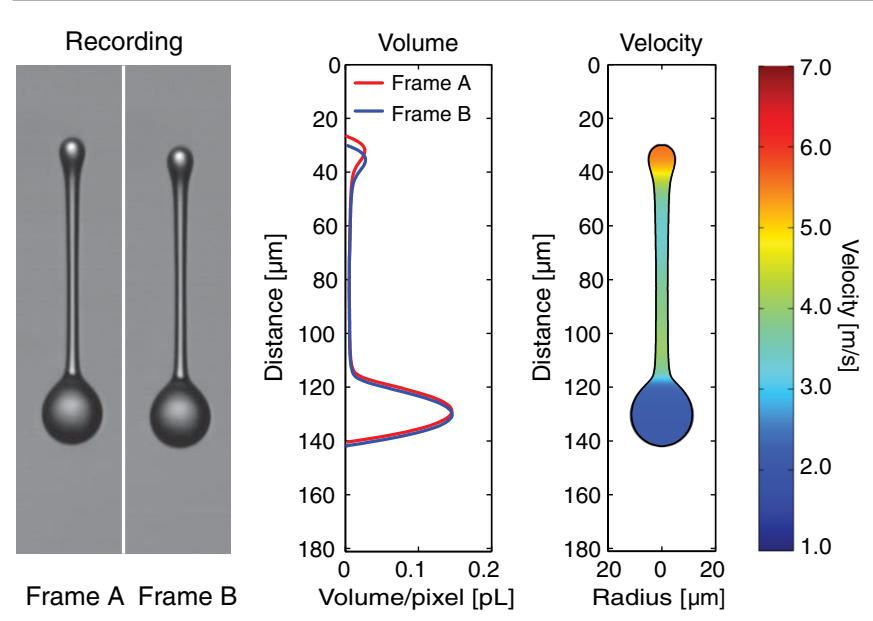

FIG. 8 (color online). The different analysis steps in the experimental procedure. Left: First two recordings of the same droplet are made, with a delay of $600 \mathrm{~ns}$ between the two frames. Middle: For both images the volume distribution over the droplet is calculated with subpixel accuracy. Right: The velocity inside the droplet is determined by calculating the displacement of the volume elements. The velocity is represented by the color scale.

counterpart. The velocity is subsequently determined by calculating the displacement of the center of mass of each element,

$$
U_{i}=\frac{\bar{x}_{A}(i)-\bar{x}_{B}(i)}{\tau_{f}} .
$$

Thus, for a given delay $t_{d}$, this method reveals the average velocity $U_{i}$ of each element $\bar{x}_{A}(i)$ throughout the droplet. This is illustrated in Fig. 8, where the left part shows the image pair, the middle part shows the volume of the droplets in the image pair, and the right part shows the calculated velocity.

\section{VALIDATION OF THE EXPERIMENTAL METHODS}

With the velocity and volume distribution known at any given time, we can also simulate the drop formation numerically. We use the velocity and volume distribution obtained from the experiment as initial condition for the numerical simulation. The drop formation is simulated in a lubrication approximation (also known as slender jet approximation); a systematic reduction of the NavierStokes equations, based on the slenderness of the liquid jet. It has been shown that this approximation gives accurate results for the evolution of a slender jet $[8,10$ $12,27,28]$. Here the previously developed discretization model by Driessen and Jeurissen [11] is used. In this discretization model the singularities, that occur at pinchoff and coalescence, are removed by adding a regularization to the surface tension term. As the regularization scales with the detail of the simulation, its influence vanishes in the limit of an infinitesimally small spatial step size. The model returns the shape, volume distribution, and the average axial velocity of the droplet, similar as we extract from the experiments.

For the validation two different cases were used. The first case is the evolution of a single droplet under normal jetting conditions, ejected at a drop on demand frequency of $10 \mathrm{kHz}$. As initial condition for the start of the numerical simulation, we use the experimentally obtained velocity and volume distribution after the droplet has detachment from the meniscus. Additionally, the density, viscosity, and surface tension of the silicone oil were used as input parameters. Figure 9 shows the evolution of the droplet for the experimental method and the numerical simulation, where we find excellent agreement. The connecting filament between tail and head remains stable during the entire contraction, i.e., it doesn't break up due to capillary forcing. Furthermore, the quantitative agreement of both magnitude

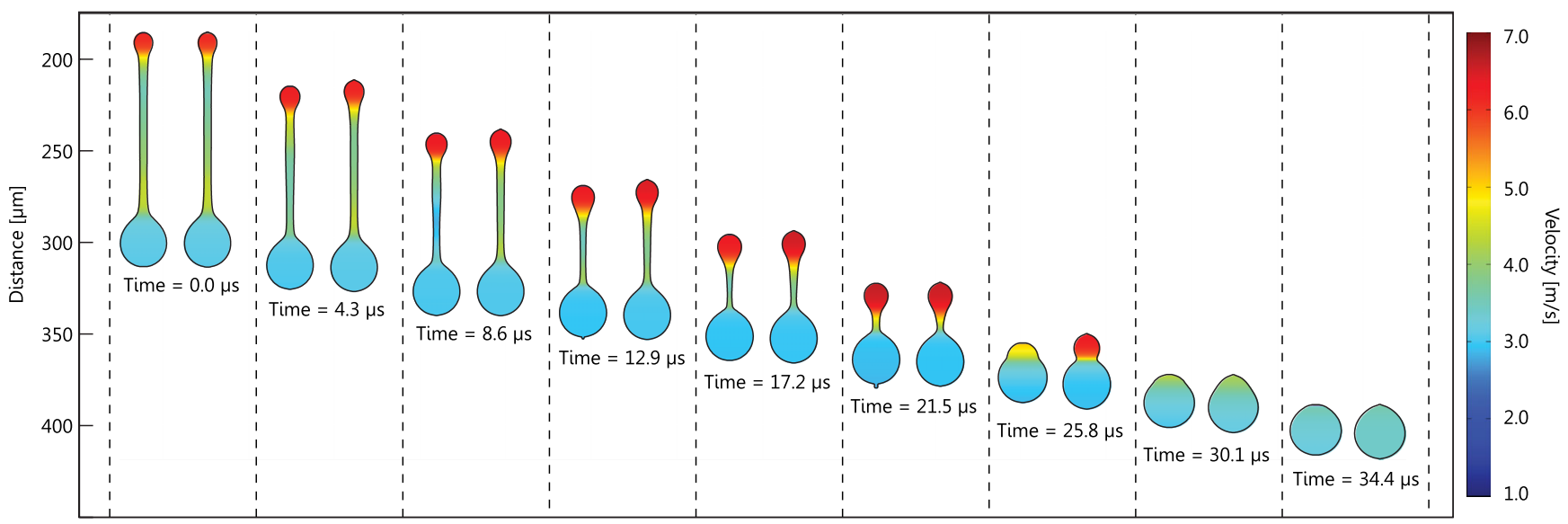

FIG. 9 (color online). Comparison between the experimental and numerical result. At different times, we show both the experimental result (left) and the numerical result (right). The experimental result is obtained from a different droplet each time, whereas the numerical droplet is calculated from the initial volume and velocity distributions from the experiment shown at $t=0 \mu \mathrm{s}$ (see also Fig. 10). The velocity is represented by the color scale. 


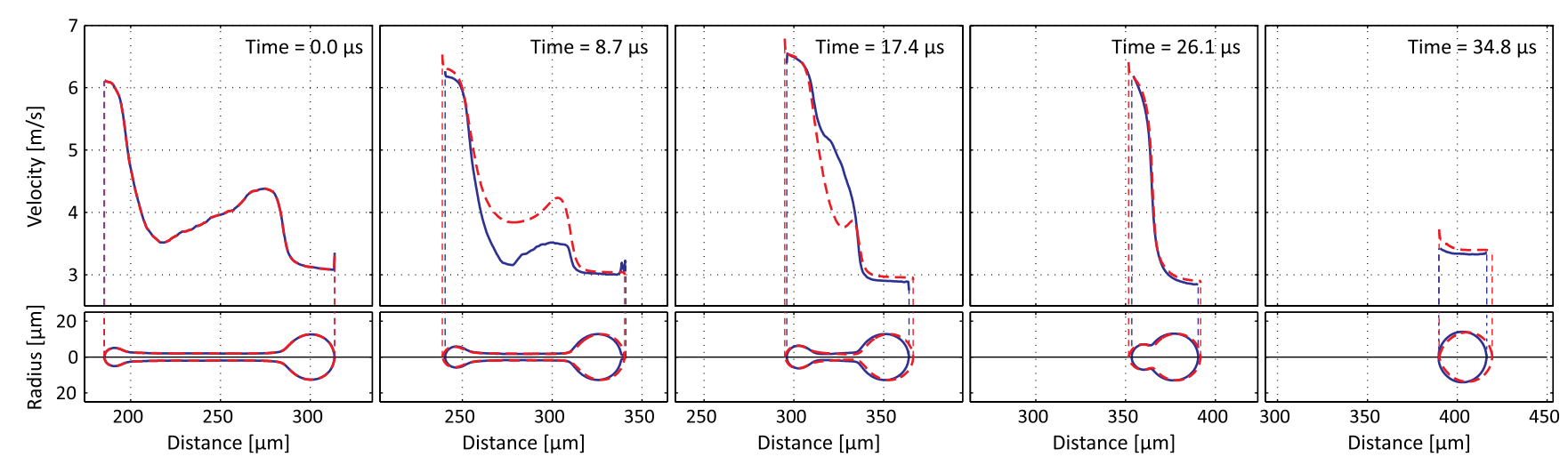

FIG. 10 (color online). Detailed comparison between the experimental result (blue) and the numerical result (dashed red) at five times instances. The top row shows the velocity distribution, whereas the bottom row shows the profiles. The experimental and the numerical profiles are nearly identical. The Rayleigh-Plateau instability causes the velocity inside the tail to vary from droplet to droplet (also visible in Fig. 11). During the contraction of the droplet there is very good agreement between the velocities of the head and tail droplet.

and distribution of the velocity are very good during the full dynamical process.

To compare the results in more detail, Fig. 10 shows the velocity and radius at five different times. For each time step we show the experimental result (solid blue line) and the numerical result (dashed red line). The experimental and the numerical profiles are nearly identical. However, there is a small distortion in the velocity in the tail of the droplet over the different experimental results. As the experimental results are obtained from a different droplet each time, the Rayleigh-Plateau instability causes the velocity inside the tail to vary from drop to drop. To support this claim, we show the experimental data of the

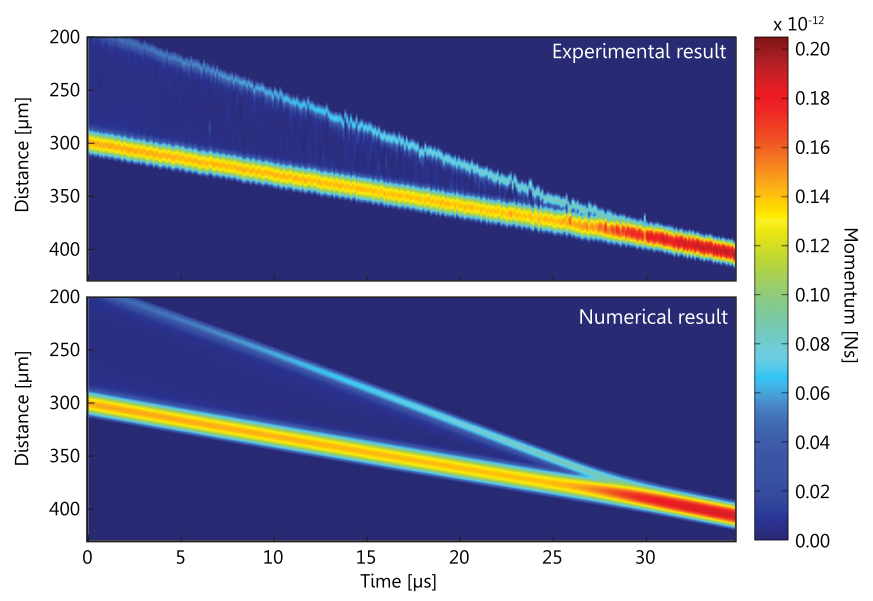

FIG. 11 (color online). The figure shows the spatial and temporal dependance of the momentum, calculated by multiplying the velocity distribution with the mass distribution. The drop flies from top to bottom. The upper figure shows the experiments, and the lower one the numerical simulation. It can be observed how the tail of the droplet is pulled towards the main drop by capillary forces. In the experimental plot it can be seen that the position of the tail and the momentum in the tail vary slightly between different droplets due to the Rayleigh breakup. This leads to the visible jitter in the curve of the tail. stroboscopic recording with a high temporal resolution in Fig. 11. It shows the momentum distribution over the droplet for all drops in the experiment (top) as compared to the numerical simulation (bottom). Here it can be observed that the perturbations on the tail of the droplet are uncorrelated between the different droplets in the experiment.

To demonstrate that the method to determine the velocity also works for separated liquid bodies, we apply it to a second case. Here two droplets are jetted from the same nozzle with a short time delay. The trailing droplet is ejected with a higher velocity, causing the two droplets to collide at approximately $350 \mu \mathrm{m}$ downstream from the nozzle. Figure 12 shows the experimental and the numerical results side by side, and again we obtain very good agreement. Again we observe small erratic behavior in the experiments. The momentum distribution as a function of time, displayed in Fig. 13, shows these disturbances more clearly. The jetting reproducibility for the case of two colliding droplets is lower than for the former case with a single droplet (Fig. 11). Still, even for this second case the overall agreement is very good.

\section{CONCLUSION AND OUTLOOK}

This work presents a novel method for quantitative analysis of the internal flow of drop formation in flight. Droplets are imaged using laser-induced fluorescence illumination, with a very short illumination time, allowing for detailed images without motion blur. Two images of a single droplet are imaged by using a dual frame setup. The images are analyzed and the volume is calculated. Here we find that the reproducibility of inkjet printing is approximately $0.13 \mathrm{pl}$ and an accuracy in the volume estimate approximately $17 \mathrm{fl}$. The reproducibility is limited and, to accurately extract the droplet dynamics, at least two images of a single droplet are required. Here we demonstrate a method to extract the one dimensional velocity inside the droplet, and validate the method with a numerical model. 


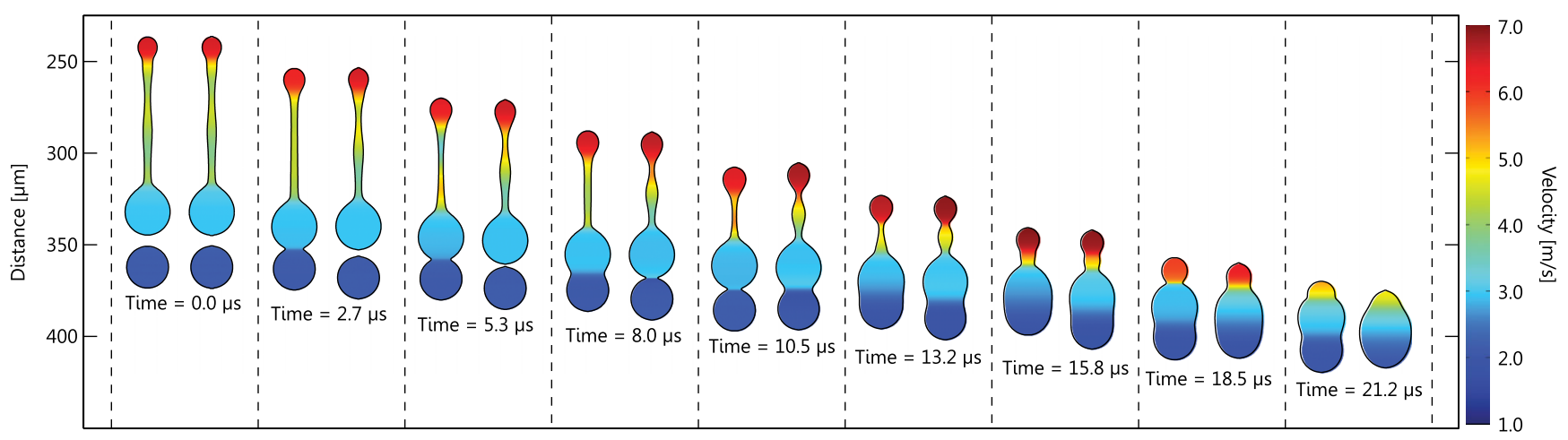

FIG. 12 (color online). Comparison between the experimental and numerical result for the case of colliding droplets. We show the experimental evolution (left) and the numerical results (right) at different times. The numerical droplet evolution is calculated from the experimental volume and velocity distributions at $t=0 \mu \mathrm{s}$. The color scale represents the velocity.

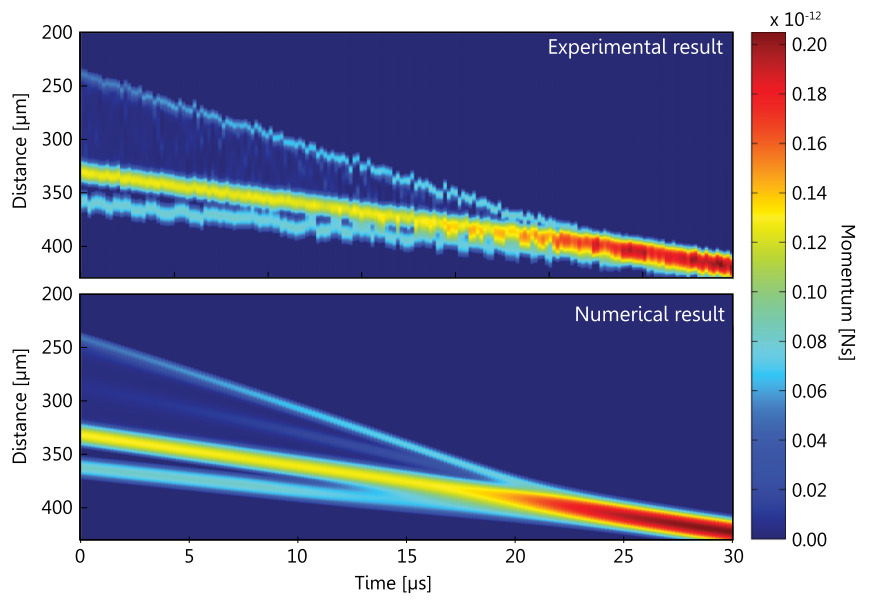

FIG. 13 (color online). The momentum distribution for the colliding droplets in experiment (top) and numerical simulation (bottom) showing overall good agreement but with an apparent lower experimental reproducibility in droplet behavior.

We find excellent agreement, confirming the applicability of the method.

The presented method is intended to study drop formation, in order to verify numerical methods, and to increase numerical utilization. This method may be extended towards drop formation in jetting of complex fluids with non-Newtonian rheology, quantitatively extracting non-Newtonian parameters from such a comparison.

With two recordings of the same droplet, it is only possible to measure its position and velocity. Technically it would be feasible to expand the setup, such that a series of images of a single droplet is captured at high spatial and temporal resolution. A third recording of the same droplet would in principle suffice to extract also the acceleration of the fluid. From the acceleration and the contour of the droplet, the forces that act on the liquid can be calculated. The capillary forces can be calculated from the droplet curvature, and therefore the local viscosity can be obtained. Thus, this method can be used as an extensometer to measure non-Newtonian properties and ink rheology at high speeds and high stretch rates encountered during inkjet drop formation.

\section{ACKNOWLEDGMENTS}

This work is part of the research programme of the Foundation for Fundamental Research on Matter (FOM), which is part of The Netherlands Organization for Scientific Research (NWO). This work is cofinanced by the Dutch ministry of economical affairs, Limburg Province, Overijssel Province, Noord-Brabant Province and the partnership region Eindhoven.

[1] H. Dong, W. W. Carr, and J. F. Morris, Visualization of drop-on-demand inkjet: Drop formation and deposition, Rev. Sci. Instrum. 77, 085101 (2006).

[2] G. D. Martin, S. D. Hoath, and I. M. Hutchings, Inkjet printing - the physics of manipulating liquid jets and drops, J. Phys. Conf. Ser. 105, 012001 (2008).

[3] H. Wijshoff, The dynamics of the piezo inkjet printhead operation, Phys. Rep. 491, 77 (2010).

[4] Hue P. Le, Progress and trends in ink-jet printing technology, J. Imaging Sci. Technol. 42, 49 (1998), http://imaging .org/IST/publications/jist/addlpages/JIST_42_1_ADD.PDF.

[5] M. Singh, H. M. Haverinen, P. Dhagat, and G. E. Jabbour, Inkjet printing process and its applications, Adv. Mater. 22, 673 (2010).

[6] J. Park, M. Hardy, S. J. Kang, K. Barton, K. Adair, D. Mukhopadhyay, C. Y. Lee, M. S. Strano, A. G. Alleyne, J. G. Georgiadis, P. M. Ferreira, and J.A. Rogers, High-resolution electrohydrodynamic jet printing, Nat. Mater. 6, 782 (2007).

[7] S. Mishra, K. L. Barton, A. G. Alleyne, P. M. Ferreira, and J. A. Rogers, High-speed and drop-on-demand printing with a pulsed electrohydrodynamic jet, J. Micromech. Microeng. 20, 095026 (2010). 
[8] J. Eggers and T.F. Dupont, Drop formation in a onedimensional approximation of the Navier-Stokes equation, J. Fluid Mech. 262, 205 (1994).

[9] C. E. Brennen, Cavitation and Bubble Dynamics (Oxford University Press, New York, 1995).

[10] W. van Hoeve, S. Gekle, J. H. Snoeijer, M. Versluis, M. P. Brenner, and D. Lohse, Breakup of diminutive Rayleigh jets, Phys. Fluids 22, 122003 (2010).

[11] T. Driessen and R. Jeurissen, A regularised one-dimensional drop formation and coalescence model using a total variation diminishing (TVD) scheme on a single Eulerian grid, Int. J. Comput. Fluid Dyn. 25, 333 (2011).

[12] E. D. Wilkes, S. D. Phillips, and O. A. Basaran, Computational and experimental analysis of dynamics of drop formation, Phys. Fluids 11, 3577 (1999).

[13] A. U. Chen, P. K. Notz, and O. A. Basaran, Computational and experimental analysis of pinch-off and scaling, Phys. Rev. Lett. 88, 174501 (2002).

[14] S. T. Thoroddsen, T. G. Etoh, and K. Takehara, High-speed imaging of drops and bubbles, Annu. Rev. Fluid Mech. 40, 257 (2008).

[15] M. Versluis, High-speed imaging in fluids, Exp. Fluids 54, 1 (2013).

[16] I. M. Hutchings, G. D. Martin, and S. D. Hoath, High speed imaging and analysis of jet and drop formation, J. Imaging Sci. Technol. 51, 438 (2007).

[17] J. de Jong, G. de Bruin, H. Reinten, M. van den Berg, H. Wijshoff, M. Versluis, and D. Lohse, Air entrapment in piezodriven inkjet printheads, J. Acoust. Soc. Am. 120, 1257 (2006).

[18] J. de Jong, R. Jeurissen, H. Borel, M. van den Berg, H. Wijshoff, H. Reinten, M. Versluis, A. Prosperetti, and D. Lohse, Entrapped air bubbles in piezo-driven inkjet printing: Their effect on the droplet velocity, Phys. Fluids 18, 121511 (2006).

[19] B. Beulen, J. de Jong, H. Reinten, M. van den Berg, H. Wijshoff, and R. van Dongen, Flows on the nozzle plate of an inkjet printhead, Exp. Fluids 42, 217 (2007).
[20] R. Jeurissen, A. van der Bos, H. Reinten, M. van den Berg, H. Wijshoff, J. de Jong, M. Versluis, and D. Lohse, Acoustic measurement of bubble size in an inkjet printhead, J. Acoust. Soc. Am. 126, 2184 (2009).

[21] J. W. Shan, D. B. Lang, and P. E. Dimotakis, Scalar concentration measurements in liquid-phase flows with pulsed lasers, Exp. Fluids 36, 268 (2004).

[22] T. Berg, J. Deppe, D. Michaelis, H. Voges, and S. Wissel, Comparison of particle size and velocity investigations in sprays carried out by means of different measurement techniques, in Proceedings of the 10th International Conference on Liquid Atomization and Spray Systems, http://www.ilasseurope.org/ICLASS/ICLASS2006/DATA/ PDF/F3-01-151.pdf.

[23] A. van der Bos, A. Zijlstra, E. Gelderblom, and M. Versluis, iLIF: illumination by laser-induced fluorescence for single flash imaging on a nanosecond timescale, Exp. Fluids 51, 1283 (2011).

[24] M. W. Davidson and M. Abramowitz, Optical microscopy, Encyclopedia of Imaging Science and Technology (Wiley, New York, 2002), Vol. 2, pp. 1106-1141 [http:// www.microscopyu.com/references/pdfs/Davidson_and_ Abramowitz_2002.pdf].

[25] C. T. Chin, C. Lancée, J. Borsboom, F. Mastik, M. E. Frijlink, N. de Jong, M. Versluis, and D. Lohse, Brandaris 128: A digital 25 million frames per second camera with 128 highly sensitive frames, Rev. Sci. Instrum. 74, 5026 (2003).

[26] M. Yu. Khomyakov, Comparative evaluation of linear edge detection methods, Pattern Recognit. Image Anal. 22, 291 (2012).

[27] X. D. Shi, M. P. Brenner, and S. R. Nagel, A cascade of structure in a drop falling from a faucet, Science 265, 219 (1994).

[28] P. K. Notz and O. A. Basaran, Dynamics and breakup of a contracting liquid filament, J. Fluid Mech. 512, 223 (2004). 